\title{
La colonoscopía detecta neoplasias avanzadas que no son diagnosticadas por la rectosigmoidoscopía
}

Use of colonoscopy to screen asymptomatic adults for colorectal cancer. Lieberman D, Weiss D, Bond J, et al. N Engl J Med 2000; 343: 162-168.

\section{Objetivo}

Determinar la prevalencia y localización de la neoplasia colónica en pacientes asintomáticos, el riesgo de neoplasia proximal avanzada en pacientes con y sin neoplasia en el colon distal y la probabilidad que la neoplasia proximal avanzada fuera detectada en base a la presencia de un adenoma en el colon distal.

\section{Diseño}

Estudio de corte transversal.

\section{Lugar}

13 centros médicos de EE.UU.

\section{Pacientes}

Se enrolaron 3196 pacientes de 50 a 75 años de edad

\section{Descripción de las pruebas y del test de referencia}

La colonoscopía (hasta el ciego) se intentó realizar a todos los pacientes incorporados. La división entre colon proximal y distal fue definida arbitrariamente por los investigadores, quedando definido como colon distal al compuesto por el rectosigma y colon descendente sin incluir el ángulo esplénico colónico, y colon proximal como aquel compuesto por el ángulo esplénico, el colon transverso y colon ascendente hasta el ciego.

De las lesiones colónicas halladas tanto en colon proximal como distal se tomaba en cuenta la lesión histológicamente más avanzada. Por ejemplo si en colon proximal se hallaba un pólipo hiperplásico y un adenoma velloso, se tomaba en cuenta la lesión del adenoma velloso y si en ese mismo paciente se encontraba en colon distal dos pólipos hiperplásicos, se consignaba que la lesión distal correspondía a pólipos hiperplásicos.

En base a esto, se determinó el riesgo de neoplasia colónica avanzada (NCA) del colon proximal en relación con las del colon distal. La NCA fue definida como: la compuesta por adenomas de $10 \mathrm{~mm}$ o más, adenomas vellosos (25\% del pólipo compuesto por adenoma velloso era considerado como tal), adenomas con alto grado de displasia, o carcinoma in situ o invasor.

\section{Medición de resultados principales}

Por medio del estudio histopatológico se evaluó a cada pólipo extirpado y se clasificó aquellos pacientes con más de un pólipo de acuerdo a la lesión más avanzada que presentaban. La lesión más avanzada tanto en el colon proximal como en el distal fue identificada separadamente para cada paciente.

\section{Resultados}

Fueron enrolados 3196 pacientes, y 3121 (97.7\%) completaron el estudio de colon. La edad media fue de 62.9 años, el $96.8 \%$ fueron hombres. De los 3121 pacientes $62.5 \%$ no presentaban neoplasias. El restante $37.5 \%$ tenían una o más lesiones neoplásicas. La NCA fue hallada en 329 pacientes (10.5\%).

La prevalencia de NCA en el colon distal fue de $7.3 \%$ (228 pacientes) y $4.1 \%$ para el proximal (128). Al limitar el colon dista al rectosigma y el proximal a todo el resto, la prevalencia NCA disminuyó al $6 \%$ en el distal (188) y aumentó al 5.4\% (169) en el proximal. Al definir colon distal como el compuesto por el rectosigma sólo el $37.9 \%$ de los pacientes con NCA presentaron un pólipo distal.

Entre los pacientes que no presentaron adenomas distales (por debajo del ángulo esplénico) el $2.7 \%$ tenía NCA, número que se incrementó a $3.7 \%$ al considerar colon distal formado por el rectosigma.

\section{Conclusión}

De acuerdo a este estudio, utilizar la colonoscopía como primer método de rastreo permite diagnosticar NCA que no serían diagnosticadas al utilizar la rectosigmoidoscopía, ya que existen neoplasias colónicas proximales en pacientes sin expresión de neoplasia en el colon distal.

Fuente de financiamiento: Programa de estudios de veterans affairs. EE.UU

\section{Comentario}

De acuerdo a estudios previos existe fuerte evidencia que muestra que el rastreo de cáncer de colon en población general mayor de 50 años disminuye la tasa de mortalidad por esta causa. 1-3 En Argentina es la tercera causa de muerte por tumores malignos, precedida por el cáncer de pulmón y de mama. Aún así no existe una estrategia única de rastreo y lo que se hace en la práctica diaria es conjugar la evidencia, los recursos disponibles y la preferencia de los pacientes.

La Fuerza de Tareas Americana recomienda realizar sangre oculta en materia fecal (SOMF) anual, sigmoidoscopía cada 5 años o ambas. De obtener un resultado positivo debe estudiarse todo el colon por medio de colonoscopía, o colon por enema doble contraste (CEDC) asociada a una rectosigmoidoscopía de no haber sido hecha previamente.

Hasta el día de hoy no existe fuerte evidencia que el rastreo por medio de la colonoscopía o el CEDC disminuya la mortalidad por cáncer de colon. A partir de este estudio, que demuestra que utilizando la sigmoidoscopía como único método de screening, se pierden de diagnosticar neoplasias proximales avanzadas, algunas de ellas en estadios previos al carcinoma e inclusive carcinomas potencialmente curables, seguramente surgirán las inquietudes de los investigadores de realizar estudios randomizados y controlados para determinar si el rastreo de cáncer de colon con colonoscopía disminuye la mortalidad por esta causa.

Dra. Carina A. Fernández [ Unidad de Medicina Familiar y Preventiva. Hospital Italiano de Buenos Aires ] 